\title{
Nun ist Mut gefragt
}

Die Revision von Kapitel 30 (Radiologie) des TARMED ist abgeschlossen. Sie dauerte drei Jahre - eine echte Meisterleistung. Wenn es in diesem Tempo weitergeht, benötigen wir nur knapp 100 Jahre, um den gesamten TARMED zu revidieren. Da kann ich uns nur viel Glück wünschen! Es besteht kein Zweifel, dass eine Revision notwendig ist. In den rund vier Jahren seit seiner Einführung kristallisierten sich die Grenzen des TARMED klar heraus: Er ist unangemessen, weil die Eckwerte für die Vergütung der Leistungen nie aktualisiert wurden. Er ist auch unangemessen, weil er nie an die Entwicklung der Weiterbildung angepasst wurde (Dignität). Wer ist für diese umfangreichen Aktualisierungsarbeiten verantwortlich? Sie werden von allen betroffenen Partnern übernommen: von uns selbst (der FMH), von der santésuisse, von der SUVA (MTK) und von H+. Vor diesem Hintergrund wurde die einfache Gesellschaft TARMED Suisse gegründet, deren einziger Zweck in der Korrektur und Aktualisierung der Tarifstruktur besteht. Eine Aufgabe, die uns jedes Jahr die Kleinigkeit von Zehntausenden von Franken kostet.

Doch wollen wir nicht den Mut aufbringen, um einzugestehen, dass die Ursache des Problems schlicht und einfach in einer unangemessenen und unzureichenden Tarifstruktur sowie in den unterschiedlichen Taxpunktwerten zwischen den Kantonen besteht? Ist es nicht höchste Zeit, dass wir den Mut aufbringen, alles wieder zur Diskussion zu stellen? Die Tarifstruktur, weil mit ihr das erklärte Ziel - die Aufwertung der Grundversorger nicht erreicht wurde. Den kantonalen Taxpunktwert, weil er auf lokaler Ebene eine immer komplexere und nicht nachvollziehbare Aufspaltung von Leistungen bedeutet, die doch im ganzen Land identisch sind. Die Grundversorger haben recht, wenn sie laut und deutlich Änderungen verlangen. Recht haben auch die Vertreter der Kantonalverbände, wenn sie gegen die derzeitigen Verhältnisse protestieren. Die Konvergenz des Taxpunktwerts im Bereich des KVG war schon immer ein Problem und ist dies nach wie vor. Weshalb will man nicht darüber sprechen? Nun ist Mut gefragt: Wollen wir einen neuen Tarif? Einen Einzelleistungstarif wie im gegenwärtigen System, der jedoch auf einem modernen Modell für die Berechnung der Vergütung und auf einer viel einfacheren Nomenklatur beruht? Wollen wir diesen Tarif selbst erarbeiten, oder warten wir lieber, bis er uns von den Versicherern oder vom Staat aufgezwungen wird? Statt bei jeder Gelegenheit zu murren und mit dem Austritt aus der FMH zu drohen, weil diese angeblich die Interessen ihrer Mitglieder nicht vertritt, und statt sich über die Möglichkeit zu streiten, einen Sprecher der Delegiertenversammlung zu ernennen, wäre es viel konstruktiver, wenn von den Vertretern der Dachorganisation der Ärzteschaft verlangt würde, eine Analyse der derzeitigen Lage durchzuführen und neue Vorschläge für die Zukunft zu formulieren. Denn wir sind weder Einfaltspinsel noch Betrüger: Dies haben mittlerweile sogar unsere Vertragspartner begriffen. Doch vielleicht fehlt uns einfach der Mut ...?!

Dr. med. Franco Muggli, Mitglied des Zentralvorstandes der FMH 